\title{
Tumoral markers in bladder cancer (Review)
}

\author{
OVIDIU BRATU ${ }^{1-3}$, DRAGOS MARCU ${ }^{1,2}$, RADU ANGHEL $^{2}$, DAN SPINU $^{1,2}$, \\ LUCIAN IORGA $^{1,2}$, IRINA BALESCU ${ }^{4}$, NICOLAE BACALBASA $^{5-7}$, CAMELIA DIACONU $^{8,9}$, \\ CORNEL SAVU ${ }^{10,11}$, CARMEN SAVU ${ }^{12}$ and ALEXANDRU CHERCIU ${ }^{2}$ \\ ${ }^{1}$ Department of Urology, 'Carol Davila' University of Medicine and Pharmacy, 020021 Bucharest; \\ ${ }^{2}$ Department of Urology, University Emergency Central Military Hospital, 010825 Bucharest; \\ ${ }^{3}$ Department of Urology, Academy of Romanian Scientists, 020021 Bucharest; ${ }^{4}$ Department of Visceral Surgery, \\ 'Ponderas' Academic Hospital, 021188 Bucharest; 5 Department of Obstetrics and Gynecology, \\ 'Carol Davila' University of Medicine and Pharmacy, 023991 Bucharest; ${ }^{6}$ Department of Visceral Surgery, \\ Center of Excellence in Translational Medicine, 'Fundeni' Clinical Institute, 022328 Bucharest; \\ ${ }^{7}$ Department of Obstetrics and Gynecology, 'I. Cantacuzino' Clinical Hospital, 030167 Bucharest; \\ ${ }^{8}$ Department of Internal Medicine, 'Carol Davila’ University of Medicine and Pharmacy, 020021 Bucharest; \\ ${ }^{9}$ Department of Internal Medicine, Clinical Emergency Hospital of Bucharest, 105402 Bucharest; \\ ${ }^{10}$ Department of Thoracic Surgery, 'Marius Nasta' National Institute of Pneumophtisiology, 050159 Bucharest; \\ ${ }^{11}$ Department of Thoracic Surgery, 'Carol Davila' University of Medicine and Pharmacy, 020021 Bucharest; \\ ${ }^{12}$ Department of Anesthesiology, 'Fundeni' Clinical Institute, 022328 Bucharest, Romania
}

Received February 10, 2021; Accepted March 12, 2021

DOI: $10.3892 / \mathrm{etm} .2021 .10205$

\begin{abstract}
Bladder tumors are frequently diagnosed urologic malignant diseases with an extremely high recurrence rate compared to other neoplastic tumors. Urothelial bladder carcinomas are mostly identified in their incipient form, as
\end{abstract}

Correspondence to: Dr Nicolae Bacalbasa, Department of Obstetrics and Gynecology, 'Carol Davila' University of Medicine and Pharmacy, 2 Dimitrie Racovita Street, 023991 Bucharest, Romania

E-mail: nicolae_bacalbasa@yahoo.ro

Abbreviations: ALCAM, activated leukocyte cell adhesion molecule; APE/Ref-1, apurinic/apyrimidinic endonuclease 1/redox factor-1; AUA, American Urological Association; $A U R K A$, aurora A kinase; cfDNA, cell-free DNA; ctDNA, circulating tumor-cell DNA; $E G F R$, epidermal growth factor receptor; EV, extracellular vesicle; exoDNA, exosomal DNA; HOTAIR, HOX transcript antisense RNA; $I Q G A P 3$, isoleucine glutamine motif-containing GTAase-activating proteins; $L A M B 3$, laminin subunit $\beta 3$; $L A M C 2$, laminin subunit $\gamma 2$; $L I N E$-1, long interspaced element-1; IncRNA, long non-voding RNA; miRNA/miR, microRNA; mRNA, messenger RNA; NDRG2, N-Myc downstream-regulated gene 2; TACSTD2, calcium-signal transducer 2; TERT, telomerase reverse transcriptase; $t R F$, transfer RNA fragment; TWIST1, Twist family BHLH transcription factor 1; $U B E 2 C$, urine ubiquitin conjugating enzyme E2 C; UCA1, circulating urothelial carcinoma antigen 1

Key words: urothelial bladder carcinoma, urinary biomarkers, non-muscle invasive, cytology, cystoscopy non-muscle invasive, but despite that, a third of them develop into aggressive recurrent disease. The diagnosis of bladder carcinoma at this moment is established using cytology and cystoscopy and is a great challenge for clinicians due to the lack of sensitivity. Urinary biomarkers could improve and enhance the diagnosis and screening techniques and determine a more accurate recurrence rate. However, bladder cancer is a heterogeneous disease and the existence of a single marker test with reduced cost is unlikely; thus, until then, the use of a panel of markers to obtain valuable information is inevitable even though suboptimal for use. To improve this deadlock, new biomarker panels should be identified and prepared to equalize the cost-efficiency balance. The present paper is a literature review concerning the most commonly used tumor markers in urinary bladder cancer as well as the most commonly encountered genetic modifications in such patients.

\section{Contents}

1. Introduction

2. Urinary biomarkers

3. Conclusions

\section{Introduction}

Bladder tumors are frequently diagnosed urologic malignant diseases with an extremely high recurrence rate compared to other neoplastic tumors. Bladder cancer types include squamous cell carcinoma, adenocarcinoma and urothelial carcinoma, the most common being transitional cell and 
urothelial bladder carcinoma representing the 10th most commonly diagnosed cancers worldwide (1). Urothelial bladder carcinomas are mostly identified in their incipient form, as non-muscle invasive, but despite that, a third will develop into aggressive recurrent disease (2). The diagnosis of bladder carcinoma at this moment is a great challenge for clinicians, as the only tools available for diagnosis and staging include: a) cytological analysis of urine, a pathologist-dependent method; b) cystoscopy and biopsy, an invasive and costly method; and c) computed tomography or magnetic resonance.

However, these tools may lead to the misdiagnosis of patients due to the lack of sensitivity by eluding micrometastatic disease staging or providing false-negative results; mistakes that could hinder the application of the correct treatment strategy for these patients (3).

Tumoral markers are a new type of investigative tool that aid clinicians to understand the tumor macroenvironment and microenvironment, to diagnose cancer earlier, improve outcomes, risk-stratify patients and apply disease-targeted therapy. However, despite the fact that the blood, tissue and urine markers for bladder have been extensively investigated in the last few years, the actual international guidelines are slowly adding them as routine management of bladder cancer (4).

The aim of this review is to present new urine biomarkers that could, in the next few years, improve the diagnosis, staging and detection of recurrence for patients with bladder cancer.

In this review, we collected the latest data from international studies concerning the urinary urothelial cancer biomarkers and their important role in diagnosis, screening and surveillance.

At this moment, the gold standard investigation used for diagnosing bladder cancer is cystoscopy with biopsy and urine cytology. Cytology is a non-invasive urinary test with a specificity of approximately $99 \%$ and sensitivity of a maximum $70 \%$ dependent on pathologist experience used for detection and surveillance of low-grade urothelial carcinoma. Yet, in the literature, there are articles that support the use of more specific urinary biomarker tests that can reduce the high cost currently associated with the management of urothelial carcinoma, provide new information regarding targeted treatment, and offer an earlier non-invasive patient-friendly diagnostic tool (5). However, bladder cancer is a heterogeneous disease and the existence of a single marker test with reduced cost is unlikely; thus, until then, we will need to use a panel of markers to obtain valuable information (6).

\section{Urinary biomarkers}

DNA methylation. DNA methylation is a biological process that develops early in carcinogenesis. It consists of the addition of methyl groups to a segment of DNA thus changing the activity affecting tumor initiation and progression (7). Urothelial bladder carcinoma is characterized by hypomethylation or hypermethylation (8). DNA methylation can be examined in tumor cells and circulating free DNA ( $c f D N A)$ fragments found in patient urine (9). Studies have shown that hypermethylated genes such as adenomatous polyposis coli $(A P C)$, glutathione S-transferase $\pi 1(G S T P 1)$ and retinoic acid receptor $\beta 2$ $(R A R b 2)$ are frequently found in the urine of urothelial carcinoma patients; thus, the methylation status of cyclin-dependent kinase inhibitor 2A ( $p 16 I N K 4 A)$, death-associated protein kinase $1(D A P K)$, ARF tumor suppressor ( $p 14 A R F), A P C$ and Ras association domain family member 1 (RASSF1A) tumor-suppressor genes have been found to be associated with the stage and grade of bladder cancer (10-12). Other studies have evaluated twist family BHLH transcription factor 1 (TWIST1) and nidogen 2 (NID2) gene methylation as well as spalt like transcription factor 3 (SALL3) and cystic fibrosis transmembrane conductance regulator (CFTR) concluding that the combination with cytology increases both the negative predictive value and sensitivity in patients with urothelial carcinoma or studied DNA methylation patterns that help distinguish non-invasive from muscle-invasive bladder cancer $(13,14)$. In addition, new studies concerning DNA methylation have recently provided evidence for superior prognostic value for bladder tumor recurrence compared with classic diagnostic tools, by analyzing SRY-box transcription factor 1 (SOX-1), interleukin-1 receptor associated kinase 3 (IRAK3) and Li-MET gene methylation grade. On the other hand, the study of SEPTIN9, Slit guidance ligand 2 (SLIT2) and heparan sulfate glucosamine 3-O-sulfotransferase 2 (HS3ST2) genes associated with alteration status of fibroblast growth factor 3 (FGFR3) could be used for urothelial carcinoma diagnosis, surveillance and risk stratification in non-invasive bladder cancer patients with $85 \%$ specificity and $98 \%$ sensitivity (13). Besides the encouraging results acquired in studies about methylated genes, the diagnostic precision of methylated DNA genes fluctuates between different research groups, so the results will need to be validated before clinical application $(15,16)$

Circulating tumor-cell DNA (ctDNA) and cell-free DNA ( $f D N A)$. ctDNA and $c f D N A$ status are additional alternatives for detecting urothelial bladder carcinoma in urine samples. ctDNA can be found in almost $80 \%$ of the patient probes. It contains a variation of alteration in DNA [180 and 200 base pairs (bp) and tumor-specific mutations], revealing the heterogeneity of tumors. On the other hand, ctDNA disappears from the urine after systemic therapy, which could impede clinical use. Moreover, cfDNA can be PCR-based analyzed from urine or blood and our supposition is that it can be used for screening or early detection of urothelial carcinoma in the future $(17,18)$.

Mutation status and microsatellite alterations. Microsatellites are short repeat sequences of 1-6 bp that form the DNA structure. Microsatellite alteration is defined as the modification in length of the microsatellite, being the result of epigenetic silencing or mutational inactivation of DNA mismatch repair genes which may initiate oncogenesis by silencing tumor-suppressor genes or disrupting other non-coding regulatory sequences. Literature reports that microsatellite alteration analysis by loss of heterozygosity methods is more sensitive than urine cytology alone (96 vs. 80\%) in the diagnosis of low-grade bladder carcinoma and low-stage bladder carcinoma, with $95 \%$ sensitivity for lower grades and $100 \%$ for CIS and other high-grade tumors (19).

On the other hand, new studies concerning mutational status have considered that FGFR3 mutational status, urinary telomerase reverse transcriptase (TERT) promoter mutations, 
and telomere length could be used to determine the high risk of recurrence of urothelial bladder carcinoma. Telomeric repeated amplification combined with FGFR3 and orthodenticle homebox 1 (OTX1) mutational status provide high sensitivity results for the diagnosis of non-muscle invasive urothelial bladder carcinoma, and high-grade T1 carcinoma and also for determining metastatic potential $(20,21)$.

A recent study using innovative sequencing technology to detect urinary tumor DNA using TERT amplification known as utDNA CAPP-Seq reached over $90 \%$ specificity. $u C A P P$-Seq outperformed the commonly used UroVysion test, cytology, and cystoscopy combined, suggesting that uCAPP-Seq is an encouraging method for early detection and follow-up of urothelial carcinoma (22).

Urinary tumor RNAs. In the last few years, research groups have studied the various RNA classes, such as microRNAs (miRs), transfer RNA fragments (tRFs), messenger RNAs (mRNAs), and long non-coding RNAs (lncRNAs), to identify a correlation among them and urothelial bladder cancer. All of them have shown the potential of being used as biomarkers (21). For example, miR values were increased in the case of patients with active carcinoma and after treatment (23).

Long non-coding RNAs (lncRNAs). IncRNAs are a subtype of RNA having a length of 200 nucleotides that regulate gene expression, certain epigenetic changes, but do not translate into protein. Fan et al studied lncRNA modifications in malignancy and proposed a role in the promotion of tumor progression and growth which received interest by the scientific community (24). Circulating urothelial carcinoma antigen 1 (UCAI) could be used as a urinary biomarker, with $80 \%$ sensitivity and approximately $90 \%$ specificity (25). Du et al proposed uc004cox.4 lncRNA as a biomarker for bladder cancer. The high value in urinary sediment may be associated with low recurrence-free survival (26). On the other hand, the long interspaced element-1 (LINE-1) retrotransposon was found to be associated with high recurrence-free and tumor-specific survival (27).

MicroRNAs (miRNAs). miRNAs are small non-coding RNAs approximately 22 nucleotides long that control gene expression by coupling to the 3 -untranslated section of their target messenger RNA (mRNA). Sethi et al support that certain miRNAs have an important role in carcinogenesis, evolution, and progression of cancer, thus urine is a source for miRNA detection having a high quantity of cell-free nucleic acid in sediments (28). However, the utility of miRNAs in the urine to diagnose urothelial carcinoma remains polemic mostly due to the reduced number of studies $(28,29)$.

Hanke et al studied miR-126 urinary levels and found that they are higher in urothelial carcinoma compared to non-malignant control patients and that miR-146a-5p could be used as a biomarker for high-grade urothelial carcinoma (30). On the other hand, low miR-200c values were found to be associated with progression of non-invasive urothelial carcinoma (20). Chen et al used a 74 miRNA panel and discovered 33 upregulated miRNAs and 41 downregulated miRNAs in urothelial cancer compared to control patients, the most important being let-7miR, miR-196a, miR-1268, miR-143,
miR-100, miR-101, miR-1 and miR-200 (31). The miR-200 was acknowledged as an epithelial-mesenchymal transition regulator in malignant cells by targeting zinc finger E-box binding homeobox 1,2 (ZEB1, ZEB2) and epidermal growth factor receptor $(E G F R)$ (32). A recent study in patients with bladder cancer found that miR-96 and miR-210 were present in urinary sediment despite the fact that the control cystoscopy was negative (33). The expression of miR-100 and miR-138 was recently considered as a prognostic biomarker in non-muscle-invasive bladder cancer (34).

In conclusion, a panel of 12 miRNAs could reduce the cystoscopy rates by $30 \%$, increasing specificity and sensitivity showing higher diagnostic performance, giving more exact information about the recurrence rate and about the aggressiveness and the stage than classic diagnostic methods and therefore may be the preferred methodology in the future. For example, an miRNA profiling urine study by NGS-derived analysis classified different carcinoma subtypes as follows. In non-invasive urothelial carcinoma G1/G2 patients, miR-205-5p upregulation was observed; in non-invasive urothelial carcinoma G3 patients, miR-21-5p, miR-106b-3p, miR-486-5p, miR-151a-3p, miR-200c-3p, miR-185-5p, miR-185-5p and miR-224-5p upregulation and miR-30c-2-5p and miR-10b-5p downregulation were observed. However, the results obtained through NGS analysis is a discovery that could be considered the perfect method of biomarker investigation using miRNAs (35).

Messenger RNAs (mRNAs). mRNAs are single-stranded RNA fragments that fit the genetic sequence of a gene and can be translated by the ribosome in the protein-producing process.

Circulating mRNAs are detectable in the urine of cancer patients, despite the fact that the majority of circulating mRNA is destroyed by the ribonuclease. Their role in intracellular protein communication reveals the intracellular activity, supposed to be used as urine biomarkers (36). For example, mRNA levels of urine ubiquitin conjugating enzyme E2 C (UBE2C) and isoleucine glutamine motif-containing GTAase-activating proteins (IQGAP3) in urine were found to be increased in urothelial carcinoma compared to control samples. Other studies of IQGAP3, concerning tumor aggressiveness and pathological grade, also conceded a high diagnostic accurateness, concluding that IQGAP3 might be used as a relevant biomarker for urothelial carcinoma in the context of microscopic or macroscopic hematuria (37). On the other hand, lower values of N-Myc downstream-regulated gene 2 (NDRG2) mRNA in the urine were found to be correlated with tumor grade and stage (38). Another important mRNA biomarker is carbonic anhydrase 9 splice variant mRNA that could increase the diagnostic performance for urothelial carcinoma with $72 \%$ specificity and $90 \%$ sensitivity (38).

Transfer RNA fragments ( $t R F s$ ). tRFs are short molecules that emerge after cleavage of mature tRNAs or the precursor transcript. tRFs are formed from 14- to -32 bases, single-stranded RNA. They are grouped into 3 principal types (tRF-1, -3 , and -5 ) and, by their cleavage site; they are divided into 5 subtypes. High levels of tRFs are found in malignancies. The first identified tRF in non-muscle invasive urothelial 
carcinoma and perhaps the most important is miR720/3007a, which has been considered a potential urine biomarker (39). Yet, there are few studies on the usage of tRFs as biomarkers.

Extracellular vesicles and exosomes. Exosomes are membrane-bound extracellular vesicles (EVs) secreted in body fluids by an endosomal compartment of cells. They have a function in intercellular communication and the transfer of active molecules (RNAs, DNA, and proteins). Exosomes in urine also contain miRNAs. Studies have shown that they are elevated in cancer patients. High levels of EVs were determined in the urine from patients with urothelial carcinoma. Andreu et al performed a study using a microarray platform and RT-PCR analysis, showing that miR-375 and miR146a were useful to identify high- and low-grade urothelial carcinoma (40). The application of nanowires anchored into a microfluidic substrate helped to determine EV collection, allowing the identification of EVs that contain miRNAs. Studies have determined that urinary exosomes have increased levels of HOX transcript antisense RNA (HOTAIR) together with other lncRNAs, such as HOX-AS-2, ANRIL and linc-RoR in patients with high-grade muscle-invasive bladder cancer (41). On the other hand, the loss of HOTAIR expression in bladder cancer cells was found to change the expression of SNA1, ZEB1, TWIST1, ZO1, laminin subunit $\beta 3$ (LAMB3), laminin subunit $\gamma 2$ (LAMC2) and MMP1 epithelial-to-mesenchymal transition genes (42). Also, proteomic examination identified calcium-signal transducer 2 (TACSTD2), a tumor-associated marker, in urinary exosomes (42). EVs can also be used to detect bladder carcinoma progression by delivering the discoidin I-like domain-containing protein-3 and protein EGF-like. In conclusion, EVs and exosomes are considered an important source of cancer biomarkers (43).

Novel urine-based biomarkers. Recent studies of urinary biomarkers have aimed to improve the diagnostic accuracy, sensitivity and specificity of urothelial carcinoma diagnosis. $B C L A-1$ and $B C L A-4$ are nuclear matrix proteins specifically targeting $\mathrm{BC}$ tissues, with no interference with infection, smoking, catheterization or cystitis. For example, in patients with hematuria, aurora A kinase $(A U R K A)$ may differentiate between normal urine vs. low-grade bladder carcinoma (44). Also, high levels of nicotinamide N-methyltransferase are present in urothelial bladder carcinoma and can help distinguish the pathologic grade (45). Apurinic/apyrimidinic endonuclease 1/redox factor-1 (APE/Ref-1) represents another novel urine-based biomarker for bladder carcinoma that could be used to establish stage, grade and recurrence (46). In addition, activated leukocyte cell adhesion molecule (ALCAM), a cell adhesion molecule, could be utilized for determining tumor stage and overall survival (47). UBC Rapid test that detects high levels of cytokeratin 8 and 18, may help to distinguish patients with high- vs. low-grade urothelial carcinoma (48). Schiffer et al claims that a conglomerate of 4 urinary fragments such as collagen $\alpha-1$ (I), collagen $\alpha-1$ (III), uromodulin and membrane-associated progesterone receptor component 1 (PGRMC1) can differentiate between non-invasive from muscle-invasive carcinoma (49). In addition, higher urinary levels of apolipoprotein A1, A2, B, C2, C3, E were found in the urine of patients with urothelial carcinoma (50). The benefits of these multi-urinary biomarker panels were useful in determining the grade of disease. Another new biomarker panels discovered in the urine was the combination of midkine and synuclein $\mathrm{G}$ or midikine, CEACAM1, ZAG2, clusterin and angiogenin that could increase the sensitivity and specificity of urinary cytology using immunoassay (51). A recent report published by Blanca et al based on protein detection of FGFR3/cyclin D3 proposed an important biomarker role of this combination, with a sensitivity of $90 \%$ and specificity of $73 \%$ in the detection of bladder cancer (52).

Moreover, metabolite panels could be used as biomarkers. A combination of N2-galacturonyl-L-lysine, indolyl acryloyl glycine, and aspartyl-glutamate allow establishment of the grade of urothelial bladder carcinoma. Furthermore, nicotinuric acid, acid trehalose, AspAspGlyTrp peptide, inosinic acid, ureidosuccinic acid, and GlyCysAlaLys peptide were found to be upregulated or downregulated in urothelial carcinoma, compared to controls probes (53). In addition, Loras et al reported the modification of phenylalanine, proline, tryptophan and arginine metabolisms revealed by ultraperformance liquid chromatography-tandem mass spectrometry in patients with urothelial bladder carcinoma (54). In conclusion, metabolomics holds great future promise and remains uninvestigated at this moment.

Urine analysis of components of the tumor microenvironment. A recent study by Wong et al identified urine derived lymphocytes as an available source of T cells in 32 patients with muscle-invasive urothelial carcinoma. $\mathrm{CD}^{+}$and $\mathrm{CD}^{+}$effector cells and regulatory $\mathrm{T}$ cells from urine accurately highlight the immune reaction of the body and map the tumor microenvironment, possibly determining the stage and status of the tumor (55). For example, an increased urine-derived lymphocyte count, such as PD-1 (PD-1 ${ }^{\text {hi }}$ ) high expression on $\mathrm{CD}^{+}$before cystectomy, was related to shorter recurrence-free survival. It was established that urine-derived lymphocyte examination is a dynamic liquid biopsy that characterizes the immune tumor microenvironment and could be used to determine the prognosis of the disease (56).

Urine-based gene mutation profile in bladder cancer diagnosis and prognosis. Recent research in utilizing urine probe as a biomarker to predict the presence of malignancy or to monitor the response of treatment that has captured research interest is the detection of genetic signatures. Ideally, early identification of urothelial carcinoma results in a better prognosis, offers a higher survival rate for patients, and could achieve a reduced recurrence rate. Thus, providing new diagnostic methods for non-invasive urothelial carcinoma will be of great practical significance in patient management. The cells from urinary residues of patients with non-invasive muscular bladder cancer have multiple gene mutational loads, and this is useful to predict recurrence after treatment. These research studies have gathered multiple reports on urinary gene mutational analysis as biomarkers of recurrence and progression after therapy. A recent study conducted by Christensen et al recognized 14 genes (152 mutation sites) in a study of 95 non-invasive muscular bladder cancer subjects and 67 control subjects. The study acknowledged genes such as FGFR3, PIK3CA and TP53 that, in comparison with the control group, the mutational rate of 14 genes was higher in the studied group (56). Non-invasive muscular bladder cancer diagnostic pattern was established by 
5 times cross-validation and had a good result, and determined all mutation sites in FGFR3, PIK3CA, TP53, STAG2, KTM2D and $A R I D I A$ genes. On the other hand, the recurrence rate was $30 \%$ among 95 patients during the monitoring period. Also, the mutation rate of TP53, PIK3CA, FGFR3, TSC1 and $E R B B 3$ in the studied group was higher than the control group. The study also analyzed the mutation sites of different genes and used it as a predictive model for urothelial carcinoma relapse, with $90 \%$ accuracy. The study established that the diagnosis based on studied gene mutations has high accuracy and could be used in early diagnosis and to determine early relapse rate. Also, recent studies found that these genes were useful to determine progression and metastatic rate in urothelial bladder carcinoma (56). Starting from the fact that urothelial bladder carcinoma is considered to have multiple genetic alterations, DNA analysis from urine is an important source for liquid biopsy (57). Lee et al studied the availability of cell-free DNA (cfDNA) and exosomal DNA (exoDNA) in urine and found that it is an important source for liquid biopsy in urothelial carcinoma (58). It analyzed deep sequencing for 9 gene targets and shallow whole-genome sequencing for detecting the variation that appears. The research group discovered 17 somatic mutations in 6 patients that appear in cancer including amplification of MDM2, CCND1, CCNE1 and ERBB2; and deletion of CDKN2A, RB1 and PTEN. In conclusion, urinary exoDNA represent another source that could be used for biopsy. Allory et al also presented a study on the prognostic relevance of genomic profiling analyzing TERT mutation frequency and correlated it with the detection of recurrences in urine and clinical outcome in patients. TERT mutations had $90 \%$ specificity, $62 \%$ sensitivity and $42 \%$ sensitivity in recurrent urothelial bladder cancer (59). Su et al recently reported on DNA methylation levels of six markers collected from 90 patients with non-invasive urothelial carcinoma. The optimum marker panel, a panel of three markers (SOX-1, Li-MET, and IRAK3) achieved a sensitivity and specificity of 86 and $80 / 97 \%$ in the testing for recurrence status, a result higher then cytology or cystoscopy (8). Also, urothelial cancers display a great genetic heterogeneity in contrast to many other types of tumors, the most frequent mutations being on the FGFR3 oncogene, that cause deregulation of the RAS-MAPK pathway producing mutations in the RAS oncogenes (HRAS, NRAS, KRAS). Undeniably, FGFR3 mutation analysis of urine has been performed and established a sensitivity of $58 \%$ for detecting bladder cancer in the last studies and could be used as a predictive tool but it needs more studies in the future to support their role.

Commercially available RNA/DNA based bladder cancer detection tests. Cxbladder is a family of urine biomarker laboratory tests optimized to diagnose the probability of having urothelial bladder. The test measures the following five genetic biomarkers linked to bladder cancer based on mRNA: i) HOXA13, which disturbs cell differentiation; ii) MDK, which alter angiogenesis in malignant cells; iii) IGFBP5, which reduces apoptosis; iv) CDC2 (CDK1), which finalizes the cell cycle, cell proliferation; and v) CXCR2 which is a marker for inflammation. Cxbladder has three different types of bladder cancer urinary tests: i) Cxbladder Triage: A test that excludes bladder cancer for patients with hematuria; ii) Cxbladder
Detect: A test that establishes the early possibility of having bladder cancer for high-risk patients; and iii) Cxbladder Monitor: A test used as a replacement for cystoscopy.

Another bladder cancer-detecting test is the uCAPP-seq test that uses the detection of cfDNA in urine sediment by measuring cell-free tumor DNA released from tumor cells. Uromonitor is a test that senses trace amounts of the FGFR3 mutation and TERT promoter, two of the most frequent alterations detected in urine. Another test is the Xpert Bladder Cancer Monitor, a test that measures the expression of five mRNA targets (ABL1, IGF2, UPK1B, CRH ANXA10) in hematuria and it is used as a negative predictive value of cystoscopy. It has recently been validated in a large cohort of non-muscle invasive bladder cancer cases with outstanding results. It can partially replace cystoscopy by excluding bladder cancer recurrence for the patients already diagnosed.

UroSEEK is a test that uses urine samples and detects the presence of modified numbers of chromosomes and mutations in 11 genes that identify the presence of bladder cancer or upper tract cancer. It is proposed to be used for early detection of bladder cancer in patients who have high risk or patients who require monitoring for recurrence. In a recent study on 570 patients, UroSEEK was positive in $83 \%$ of those who had urothelial carcinoma. Combined with cytology, the test identified $95 \%$ of patients who had bladder cancer. For upper tract urothelial cancer, $75 \%$ of the patients tested positive. Also, it had good results for detecting the recurrences for the patients already diagnosed. Compared with cytology, the test detected $67 \%$ of cases while cytology detected nothing (60).

The UroMuTERT is another test, capable of detecting urothelial cancer. It is based on analyzing two highly recurrent mutations in the telomerase reverse transcriptase (TERT) gene, mutations that are present in the urothelial tumor and can easily be detected in the urine of patients. The test was developed to be sensitive to low levels of TERT mutations in urine sediment and has demonstrated excellent specificity and specificity for the discovery of urothelial cancer, greater than urine cytology for the detection of low-grade early-stage carcinoma (61).

Classic biomarkers approved for clinical practice. Cystoscopy and urine cytology are the gold methods for the diagnosis and surveillance of bladder cancer, but the role of new urinary biomarker testing that could complete with or replace these two forms of examination is not well established. Biomarkers are useful in particular situations, such as to improve the atypical cytology results, to analyze the bacillus Calmette-Guerin instillations, to identify high risk patients or recurrence rate according to (AUA) American Urological Association guidelines. Some biomarkers have been accepted for clinical practice in the last 20 years. NMP22, immunoCyt (uCyt+), BTA-TRAK, BTA-STA and UroVysion have been approved by authorities for urothelial carcinoma diagnosis and surveillance. Thus the approval of urinary tumor markers has transformed the urologist tactic to diagnose urothelial carcinoma. For example, UroVysion, NMP22 and BTA are replacing urine cytology for detecting urothelial carcinoma. ImmunoCyt, on the other hand, has achieved best results combined with urine cytology enhancing the specificity and sensitivity of 78 and $90 \%$. Studies that analyzed the SEER-Medicare database highlights the fact that urinary biomarker testing has increased in the last years 
leading the urological community to use new non-invasive way of monitoring bladder cancer despite the lack of guideline endorsement, confusion regarding the usefulness, cost concerns, reimbursement changes, logistical issues and availability (62).

\section{Conclusions}

Despite the overabundance of studies investigating the role of urinary biomarkers in urothelial bladder cancer with an incredible rate of data, none of the studies has reached equilibrium between cost, relevance, the worldwide spread of techniques; thus, it will take a few more years to reach the popularity of cystoscopy and cytology $(63,64)$.

\section{Acknowledgements}

Not applicable.

\section{Funding}

No funding was received.

\section{Availability of data and materials}

All information provided in this review is documented by relevant references.

\section{Authors' contributions}

$\mathrm{OB}$ and $\mathrm{AC}$ had the initial idea for the project, developed the project, wrote the manuscript and provided consultation, DS, IB, CoS, and NB developed the project, collect the data and were major contributors to writing the manuscript. NB provided consultation to the manuscript. LI, RA, DM, and CaS collected the data and wrote the manuscript and CD provided consultation to the manuscript and supervised the project. All authors read and approved the final manuscript.

\section{Ethics approval and consent to participate}

Not applicable.

\section{Patient consent for publication}

Not applicable.

\section{Competing interests}

There are no competing interests to declare regarding this study.

\section{References}

1. Sung H, Ferlay J, Siegel RL, Leversanne M, Soerjomataram I, Jemal A and Bray F: Global cancer statistics 2020: GLOBOCAN estimates of incidence and mortality worldwide for 36 cancer in 185 countries. CA Cancer J Clin Feb 4, 2021 (Epub ahead of print). doi: $10.3322 /$ caac. 21660 .

2. Sylvester RJ, van der Meijden AP, Oosterlinck W, Witjes JA, Bouffioux C, Denis L, Newling DW and Kurth K: Predicting recurrence and progression in individual patients with stage Ta T1 bladder cancer using EORTC risk tables: A combined analysis of 2596 patients from seven EORTC trials. Eur Urol 49: 466-477, 2006
3. van Rhijn BW, Burger M, Lotan Y, Solsona E, Stief CG, Sylvester RJ, Witjes JA and Zlotta AR: Recurrence and progression of disease in non-muscle-invasive bladder cancer: From epidemiology to treatment strategy. Eur Urol 56: 430-442, 2009.

4. Tilki D, Burger M, Dalbagni G, Grossman HB, Hakenberg OW, Palou J, Reich O, Rouprêt M, Shariat SF and Zlotta AR: Urine markers for detection and surveillance of non-muscle invasive bladder cancer. Eur Urol 60: 484-492, 2011.

5. Narayan VM, Adejoro O, Schwartz I, Ziegelmann M, Elliott S and Konety BR: The prevalence and impact of urinary marker testing in patients with bladder cancer. J Urol 199: 74-80, 2018.

6. Bumbu GA, Berechet MC, Pop OL, Nacer K, Bumbu G, Maghiar OA, Bratu OG, Stefanescu ML, Pantis C and Bumbu BA: Primary malignant melanoma of the bladder-case report and literature overview. Rom J Morphol Embryol 60: 287-292, 2019.

7. Witjes JA, Morote J, Cornel EB, Gakis G, van Valenberg FJP, Lozano F, Sternberg IA, Willemsen E, Hegemann ML, Paitan Y and Leibovitch I: Performance of the bladder EpiCheck ${ }^{\mathrm{TM}}$ methylation test for patients under surveillance for non-muscle-invasive bladder cancer: Results of a Multicenter, Prospective, Blinded Clinical Trial. Eur Urol Oncol 1: 307-313, 2018.

8. Su SF, de Castro Abreu AL, Chihara Y, Tsai Y, Andreu-Vieyra C, Daneshmand S, Skinner EC, Jones PA, Siegmund KD and Liang G: A panel of three markers hyper- and hypomethylated in urine sediments accurately predicts bladder cancer recurrence. Clin Cancer Res 20: 1978-1989, 2014.

9. Goessl C, Müller M, Straub B and Miller K: DNA alteration in body fluids as molecular tumor markers for urological malignancies. Eur Urol 41: 668-676, 2002.

10. Pu RT, Laitala LE and Clark DP: Methylation profiling of urothelial carcinoma in bladder biopsy and urine. Acta Cytol 50: 499-506, 2006

11. Hauser S, Kogej M, Fechner G, VON Pezold J, Vorreuther R, Lummen G, Muller SC and Ellinger J: Serum DNA hypermethylation in patients with bladder cancer: Results of a prospective multicenter study. Anticancer Res 33: 779-784, 2013.

12. Pietrusinski M, Kȩpczyński Ł, Jedrzejczyk A, Borkowska E, Traczyk-Borszynska M, Constantinou M, Kauzewski B and Borowiec M: Detection of bladder cancer in urine sediments by a hypermethylation panel of selected tumor suppressor genes. Cancer Biomark 18: 47-59, 2017.

13. van der Heijden AG, Mengual L, Ingelmo-Torres M, Lozano JJ, van Rijt-van de Westerlo CCM, Baixauli M, Geavlete B, Moldoveanud C, Ene C, Dinney CP, et al: Urine cell-based DNA methylation classifier for monitoring bladder cancer. Clin Epigenetics 10: 71, 2018.

14. Roperch JP, Grandchamp B, Desgrandchamps F, Mongiat-Artus P, Ravery V, Ouzaid I, Roupret M, Phe V, Ciofu C, Tubach F, et al: Promoter hypermethylation of HS3ST2, SEPTIN9 and SLIT2 combined with FGFR3 mutations as a sensitive/specific urinary assay for diagnosis and surveillance in patients with low or high-risk non-muscle-invasive bladder cancer. BMC Cancer 16: 704, 2016.

15. Peng M, Chen C, Hulbert A, Brock MV and Yu F: Non-blood circulating tumor DNA detection in cancer. Oncotarget 8: 69162-69173, 2017.

16. Bosschieter J, Lutz C, Segerink LI, Vis AN, Zwarthoff EC, A van Moorselaar RJ, van Rhijn BW, Heymans MW, Jansma EP, Steenbergen RD and Nieuwenhuijzen JA: The diagnostic accuracy of methylation markers in urine for the detection of bladder cancer: A systematic review. Epigenomics 10: 673-687, 2018.

17. Brisuda A, Pazourkova E, Soukup V, Horinek A, Hrbacek J, Capoun O, Svobodova I, Pospisilova S, Korabecna M, Mares J, et al: Urinary Cell-Free DNA quantification as Non-Invasive biomarker in patients with bladder cancer. Urol Int 96: 25-31, 2016

18. Qin Z, Ljubimov VA, Zhou C, Tong Y and Liang J: Cell-free circulating tumor DNA in cancer. Chin J Cancer 35: 36, 2016.

19. Seripa D, Parrella P, Gallucci M, Gravina C, Papa S, Fortunato P, Alcini A, Flammia G, Lazzari M and Fazio VM: Sensitive detection of transitional cell carcinoma of the bladder by microsatellite analysis of cells exfoliated in urine. Int J Cancer 95: 364-369, 2001.

20. Wiklund ED, Gao S, Hulf T, Sibbritt T, Nair S, Costea DE, Villadsen SB, Bakholdt V, Bramsen JB, Sørensen JA, et al: MicroRNA alterations and associated aberrant DNA methylation patterns across multiple sample types in oral squamous cell carcinoma. PLoS One 6: e27840, 2011.

21. Bryzgunova OE and Laktionov PP: Extracellular nucleic acids in urine: Sources, structure, diagnostic potential. Acta Naturae 7: 48-54, 2015. 
22. Dudley JC, Schroers-Martin J, Lazzareschi DV, Shi WY, Chen SB, Esfahani MS, Trivedi D, Chabon JJ, Chaudhuri AA, Stehr H, et al: Detection and surveillance of bladder cancer using urine tumor DNA. Cancer Discov 9: 500-509, 2019.

23. Sasaki H, Yoshiike M, Nozawa S, Usuba W, Katsuoka Y, Aida K, Kitajima K, Kudo H, Hoshikawa M, Yoshioka Y, et al: Expression level of urinary MicroRNA-146a-5p is increased in patients with bladder cancer and decreased in those after transurethral resection. Clin Genitourin Cancer 14: e493-e499, 2016.

24. Fan Y, Shen B, Tan M, Mu X, Qin Y, Zhang F and Liu Y: Long non-coding RNA UCA1 increases chemoresistance of bladder cancer cells by regulating Wnt signaling. FEBS J 281: 1750-1758, 2014.

25. Peter S, Borkowska E, Drayton RM, Rakhit CP, Noon A Chen W and Catto JW: Identification of differentially expressed long noncoding RNAs in bladder cancer. Clin Cancer Res 20 5311-5321, 2014.

26. Du L, Duan W, Jiang X, Zhao L, Li J, Wang R, Yan S, Xie Y, Yan K, Wang Q, et al: Cell-free lncRNA expression signatures in urine serve as novel non-invasive biomarkers for diagnosis and recurrence prediction of bladder cancer. J Cell Mol Med 22: 2838-2845, 2018.

27. Neuhausen A, Florl AR, Grimm MO and Schulz WA: DNA methylation alterations in urothelial carcinoma. Cancer Biol Ther 5: 993-1001, 2006

28. Sethi S, Sethi S and Bluth MH: Clinical implication of microRNAs in molecular pathology: An update for 2018. Clin Lab Med 38: 237-251, 2018.

29. Xiao S, Wang $J$ and Xiao N: MicroRNAs as noninvasive biomarkers in bladder cancer detection: A diagnostic metaanalysis based on qRT-PCR data. Int J Biol Markers 31: e276-e285, 2016

30. Hanke M, Hoefig K, Merz H, Feller AC, Kausch I, Jocham D, Warnecke JM and Sczakiel G: A robust methodology to study urine microRNA as tumor marker: microRNA-126 and microRNA-182 are related to urinary bladder cancer. Urol Oncol 28: 655-661, 2010.

31. Chen YH, Wang SQ, Wu XL, Shen M, Chen ZG, Chen XG, Liu YX, Zhu XL, Guo F, Duan XZ, et al: Characterization of microRNAs expression profiling in one group of Chinese urothelial cell carcinoma identified by Solexa sequencing. Urol Oncol 31: 219-227, 2013.

32. Braicu C, Cojocneanu-Petric R, Chira S, Truta A, Floares A Petrut B, Achimas-Cadariu P and Berindan-Neagoe I: Clinical and pathological implication of miRNA in bladder cancer. Int J Nanomedicine 10: 791-800, 2015.

33. Eissa S, Matboli M, Essawy NO and Kotb YM: Integrative functional genetic-epigenetic approach for selecting genes as urine biomarkers for bladder cancer diagnosis. Tumour Biol 36 : 9545-9552, 2015.

34. Bianca A, Sanchez-Gonzalez A, Requena MJ, Carrasco-Valiente J, Gomez-Gomez E, Cheng L, Cimadamore A, Montironi R and Lopez-Beltran A: Expression of miR-100 and miR-138 as prognostic biomarkers in non-muscle-invasive bladder cancer. APMIS 127: 545-553, 2019.

35. Pardini B, Cordero F, Naccarati A, Viberti C, Birolo G, Oderda M, Di Gaetano C, Arigoni M, Martina F, Calogero RA, et al: MicroRNA profiles in urine by next-generation sequencing can stratify bladder cancer subtypes. Oncotarget 9: 20658-20669, 2018.

36. Hofbauer SL, de Martino M, Lucca I, Haitel A, Susani M, Shariat SF and Klatte T: A urinary microRNA (miR) signature for diagnosis of bladder cancer. Urol Oncol 36: 531.e1-531.e8, 2018.

37. Kim WT, Kim YH, Jeong P, Seo SP, Kang HW, Kim YJ, Yun SJ, Lee SC, Moon SK, Choi YH, et al: Urinary cell-free nucleic acid IQGAP3: A new non-invasive diagnostic marker for bladder cancer. Oncotarget 9: 14354-14365, 2018.

38. Zhang M, Ren B, Li Z, Niu W and Wang Y: Expression of N-Myc downstream-regulated gene 2 in bladder cancer and its potentia utility as a urinary diagnostic biomarker. Med Sci Monit 23: 4644-4649, 2017.

39. Armstrong DA, Green BB, Seigne JD, Schned AR and Marsit CJ: MicroRNA molecular profiling from matched tumor and bio-fluids in bladder cancer. Mol Cancer 14: 194, 2015.

40. Andreu Z, Otta Oshiro R, Redruello A, Lopez-Martin S, Gutiérrez-Vázquez C, Morato E, Marina AI, Olivier Gómez C and Yáñez-Mó M: Extracellular vesicles as a source for non-invasive biomarkers in bladder cancer progression. Eur J Pharm Sci 98: 70-79, 2017.
41. Berrondo C,Flax J, Kucherov V, Siebert A, Osinski T, Rosenberg A, Fucile C, Richheimer S and Beckham CJ: Expression of the Long Non-Coding RNA HOTAIR correlates with disease progression in bladder cancer and is contained in bladder cancer patient urinary exosomes. PLoS One 11: e0147236, 2016.

42. Chen CL, Lai YF, Tang P, Chien KY, Yu JS, Tsai CH, Chen HW, Wu CC, Chung T, Hsu CW, et al: Comparative and targeted proteomic analyses of urinary microparticles from bladder cancer and hernia patients. J Proteome Res 11: 5611-5629, 2012.

43. Beckham CJ, Olsen J, Yin PN, Wu CH, Ting HJ, Hagen FK, Scosyrev E, Messing EM and Lee YF: Bladder cancer exosomes contain EDIL-3/Del1 and facilitate cancer progression. J Urol 192: 583-592, 2014.

44. de Martino M, Shariat SF, Hofbauer SL, Lucca I, Taus C, Wiener HG, Haitel A, Susani M and Klatte T: Aurora A kinase as a diagnostic urinary marker for urothelial bladder cancer. World J Urol 33: 105-110, 2015

45. Pozzi V, Di Ruscio G, Sartini D, Campagna R, Seta R, Fulvi P, Vici A, Milanese G, Brandoni G, Galosi AB, et al: Clinical performance and utility of NNMT-based urine test for bladder cancer. Int J Biol Markers 33: 94-101, 2018.

46. Choi S, Shin JH, Lee YR, Joo HK, Song KH, Na YG, Chang SJ, Lim JS and Jeon BH: Urinary APE1/Ref-1: A potential bladder cancer biomarker. Dis Markers 2016: 7276502, 2016.

47. Rosso O, Piazza T, Bongarzone I, Rosello A, Mezzanzanica D, Canevari S, Orengo AM, Puppo A, Ferrini S and Fabbi M: The ALCAM shedding by metalloprotease ADAM17/TACE is involved in motility of ovarian carcinoma cells. Mol Cancer Res 5: 1246-1253, 2007.

48. Ecke TH, Weiß S, Stephan C, Hallmann S, Barski D, Otto T and Gerullis H: UBC ${ }^{\circledast}$ Rapid Test for detection of carcinoma in situ for bladder cancer. Tumour Biol 39: 1010428317701624, 2017.

49. Schiffer E, Vlahou A, Petrolekas A, Stravodimos K, Tauber R, Geschwend JE, Neuhaus J, Stolzenburg JU, Conaway MR, Mischak $\mathrm{H}$ and Theodorescu D: Prediction of muscle-invasive bladder cancer using urinary proteomics. Clin Cancer Res 15: 4935-4943, 2009

50. Chen YT, Chen CL, Chen HW, Chung T, Wu CC, Chen CD, Hsu CW, Chen MC, Tsui KH, Chang PL, et al: Discovery of novel bladder cancer biomarkers by comparative urine proteomics using iTRAQ technology. J Proteome Res 9: 5803-5815, 2010.

51. Soukup V, Kalousova M, Capoun O, Sobotka R, Breyl Z, Pesl M, Zima T and Hanus T: Panel of urinary diagnostic markers for non-invasive detection of primary and recurrent urothelial urinary bladder carcinoma. Urol Int 95: 56-64, 2015

52. Blanca A, Requena MJ, Alvarez J, Cheng L, Montironi R, Raspollini MR, Reymundo C and Lopez-Beltran A: FGFR3 and Cyclin D3 as urine biomarkers of bladder cancer recurrence. Biomark Med 10: 243-253, 2016.

53. Shen C, Sun Z, Chen D, Su X, Jian J, Li G, Lin B and Yan J: Developing urinary metabolomic signatures as early bladder cancer diagnostic markers. OMICS 19: 1-11, 2015.

54. Loras A, Trassierra M, Sanjuan-Herraez D, Martinez-Bisbal MC, Castell JV, Quintas G and Ruiz-Cerda JL: Bladder cancer recurrence surveillance by urine metabolomics analysis. Sci Rep 8: 9172,2018

55. Wong YNS, Joshi K, Khetrapal P, Ismail M, Reading JL, Sunderland MW, Georgiou A, Furness AJS, Ben Aissa A, Ghorani E, et al: Urine-derived lymphocytes as a non-invasive measure of the bladder tumor immune microenvironment. J Exp Med 215: 2748-2759, 2018.

56. Christensen E, Birkenkamp-Demtroder K, Nordentoft I, Hoyer S, van der Keur K, van Kessel K, Zwarthoff E, Agerbaek M, Orntoft TF, Jensen JB and Dyrskjøt L: Liquid biopsy analysis of FGFR3 and PIK3CA hotspot mutations for disease surveillance in bladder cancer. Eur Urol 71: 961-969, 2017.

57. Gormally E, Caboux E, Vineis $\mathrm{P}$ and Hainaut P: Circulating free DNA in plasma or serum as biomarker of carcinogenesis: Practical aspects and biological significance. Mutat Res 635: 105-117, 2007.

58. Lee J, McKinney KQ, Pavlopoulos AJ, Niu M, Kang JW, Oh JW, Kim KP and Hwang S: Altered proteome of extracellular vesicles derived from bladder cancer patients urine. Mol Cells 41: 179-187, 2018.

59. Allory Y, Beukers W, Sagrera A, Flandez M, Marques M, Marquez M, van der Keur KA, Dyrskjot L, Lurkin I, Vermeij M, et al: Telomerase reverse transcriptase promoter mutations in bladder cancer: High frequency across stages, detection in urine, and lack of association with outcome. Eur Urol 65: 360-366, 2014 
60. Springer SU, Chen CH, Rodriguez Pena MDC, Li L, Douville C, Wang Y, Cohen JD, Taheri D, Silliman N, Schaefer J, et al: Non invasive detection of urothelial cancer through the analysis of driver gene mutation and aneuploidy. Elife 7: e32143, 2018.

61. Avogbe PH, Manel A, Vian E, Durand G, Forey N, Voegele C, Zvereva M, Hosen MI, Meziani S, De Tilly B, et al: Urinary TERT promoter mutation as non-invasive biomarkers for the comprehensive detection of urothelial cancer. EBioMedicine 44: 431-438, 2019.
62. Lozano F, Raventos CX, Carrion A, Trilla E and Morote J: Current status of genetic urinary biomarkers for surveillance of non-muscle invasive bladder cancer: A systematic review. BMC Urol 20: 99, 2020

63. Boda D: Cellomics as integrative omics for cancer. Curr Proteomics 10: 237-245, 2013.

64. Neagu M, Constantin C, Tanase C and Boda D: Patented biomarker panels in early detection of cancer. Recent Pat Biomark 1: 10-14, 2011. 